\title{
Estudo ecológico de séries temporais sobre a mortalidade por câncer de pele na Região Sul do Brasil
}

Ecological time series study on mortality in skin cancer in Southern of Brazil

\author{
Vinicius Pinheiro Nunes', Angela Isabel dos Santos Dullius", \\ Vanessa Siqueira Peres da Silva"', Michael Gonçalves da Silva', \\ Angela Pellegrin Ansuj ${ }^{v}$
}

\begin{abstract}
RESUMO
O objetivo deste estudo foi analisar a tendência da mortalidade e ajustar um modelo de séries temporais para as doenças neoplásicas de pele, no período de 1996 a 2012, na região sul do Brasil. Para tanto, realizou-se um estudo ecológico de séries temporais, utilizando dados de óbitos trimestrais. O Modelo $\operatorname{ARIMA}(4,1,0)$ foi o que teve melhor ajuste aos critérios adotados e validados com os dados de 2013. Constatou-se nesse estudo que há um aumento do número de óbitos por câncer de pele ao longo do tempo, as quais podem ser previstas pelo modelo determinado, e essas previsões podem auxiliar no planejamento de políticas públicas de intervenção e/ou prevenção do câncer de pele junto à população da Região Sul do Brasil.
\end{abstract}

Palavras-chave: ARIMA; Modelo Box-Jenkis; Neoplasias Cutâneas.

\begin{abstract}
The aim of this study was to analyze the mortality trend and to adjust a time series model for neoplastic skin diseases, from 1996 to 2012, in southern Brazil. To do so, conduct an ecological time series study using quarterly death data. The ARIMA Model $(4,1,0)$ was the one that had the best fit for the date adopted and validated with date from 2013. It can be seen in this study that there is an increase in the number of deaths from skin cancer over time, How Which can be approved by the given model, and which can be helpful in planning public policies for intervention and / or prevention of skin cancer among the population of Southern Brazil.
\end{abstract}

Keywords: ARIMA; Modelo Box-Jenkis; Skin Neoplasms.

\footnotetext{
' Universidade Federal de Santa Maria, Santa Maria, Brasil. E-mail: viniciusnunes92@hotmail.com.

"Universidade Federal de Santa Maria, Santa Maria, Brasil. E-mail: angeladullius@gmail.com.

III Universidade Federal de Santa Maria, Santa Maria, Brasil. E-mail: dexvanessa@gmail.com.

IV Universidade Federal de Santa Maria, Santa Maria, Brasil. E-mail: michael_gsilva@yahoo.com.br.

v Universidade Federal de Santa Maria, Santa Maria, Brasil. E-mail: angelaansuj@yahoo.com.
} 


\section{INTRODUÇÃO}

O câncer destaca-se como a principal causa de mortalidade entre países que apresentam economia desenvolvida e como segunda principal causa entre aqueles que estão em desenvolvimento (JEMAL, 2011). No Brasil, essa doença tem ganhado importante notoriedade devido ao seu caráter epidemiológico, tornando-se alvo de diversas discussões em órgãos relacionados ao governo (INSTITUTO NACIONAL DE CÂNCER JOSÉ ALENCAR GOMES DA SILVA, 2014). Ainda assim, por se tratar de um relevante problema de saúde pública, quaisquer assuntos relacionados a essa temática devem ser mais bem discutidos, principalmente no que se refere aos tipos de câncer que mais acometem a população brasileira.

O Instituto Nacional de Câncer José Alencar Gomes da Silva (INCA) é o órgão brasileiro responsável por desenvolver e implementar políticas de controle e prevenção dessa enfermidade. De acordo com estimativas realizadas por esse órgão, os tipos de câncer/neoplasias que apresentarão maior incidência em 2015 serão os relacionados a pele, representando mais de $30 \%$ do total de novos casos esperados (INSTITUTO NACIONAL DE CÂNCER JOSÉ ALENCAR GOMES DA SILVA, 2014). Em razão dessa projeção elevada, fica evidente a necessidade de implementar novas políticas para controlar ou até mesmo reduzir esse número.

As neoplasias malignas de Pele manifestam-se de diferentes formas em seres humanos há milhares de anos (MARKS; KOPF, 1995). No sentido amplo, há duas formas de manifestação dessa doença: o não melanoma, tipo mais frequente, porém menos agressivo; e o melanoma, tipo menos frequente porém mais agressivo que apresenta chances de cura de até 90\% quando tratado precocemente (RHODES et al., 1987; SBD, 2014). Em diagnóstico tardio, as chances de metástase dos melanomas são bastante altas, devido a isso as chances de cura diminuem, podendo levar fatalmente o indivíduo ao óbito (SBD, 2014). Por isso a importância de iniciativas, não somente no sentido de diagnosticar prematuramente esses tipos de neoplasias, mas sobretudo no que se refere a prevenção de novos casos.

A região brasileira de maior incidência de neoplasias malignas de pele é a Região Sul. Segundo dados do Departamento de Informática do Sistema Único de Saúde 
(DATASUS), essa alta incidência resultou em 10549 óbitos relacionados à pele, entre 1996 e 2012 (DATASUS, 2014). Cronologicamente, a variação trimestral desses óbitos foi de 98 a 125 óbitos entre 1996 e 1999, 110 a 157 óbitos entre 2000 e 2003, 136 a 210 óbitos entre 2004 e 2007, e 160 a 230 entre 2008 e 2012 (DATASUS, 2014). A partir desses dados é possível observar o crescente número de óbitos ocorridos ao longo do tempo, explicitando a necessidade de realização de estudos e pesquisas nessa região.

Diante disso, esse estudo tem como objetivo estimar um modelo explicativo para o comportamento da série trimestral do número de óbitos por câncer de pele na Região Sul do Brasil, entre 1996 e 2013, por meio de técnicas estatísticas, com o intuito de se obter resultados que possam ser úteis para alertar a população e órgãos competentes quanto a necessidade de implementar melhorias nas políticas públicas voltadas a prevenção e controle dessa doença.

\section{MÉTODOS}

Trata-se de um estudo ecológico de séries temporais sobre o número de óbitos trimestrais por câncer de pele, ocorridos na Região Sul do Brasil, na qual abrange os Estados do Rio Grande do Sul (RS), Santa Catarina (SC) e Paraná (PR). Foram considerados os óbitos entre primeiro trimestre de 1996 ao quarto trimestre de 2013, sendo os dados de 1996 a 2012 para a obtenção do modelo explicativo, e os dados de 2013 na validação deste modelo.

Foram todos os 10549 óbitos que apresentaram como causa básica o melanoma maligno de pele ou outros tipos de neoplasias malignas de pele. Os casos relacionados a essas terminologias incluem todos os subtipos registrados com os códigos C43 e C44 da Classificação Estatística Internacional de Doenças e Problemas Relacionados com a Saúde, Décima Revisão (CID-10), conforme apresentados no Quadro 1. Apenas os óbitos com idade ignorada foram excluídos da análise. A coleta das informações foi realizada através do Sistema de Informações sobre Mortalidade do Ministério da Saúde (SIM), disponibilizado pelo DATASUS. 
Quadro 1 - Descrição dos tipos de melanomas e outras (os) neoplasias (tumores) malignas (os) da pele (C43-C44) de acordo com a CID-1

\begin{tabular}{|l|l|}
\hline Códigos & Melanomas malignos da pele (C43) \\
\hline C43.0 & Melanoma maligno do lábio \\
\hline C43.1 & Melanoma maligno da pálpebra, incluindo as comissuras palpebrais \\
\hline C43.2 & Melanoma maligno da orelha e do ducto auditivo externo \\
\hline C43.3 & Melanoma maligno de outras partes e partes não especificadas da face. \\
\hline C43.4 & Melanoma maligno do couro cabeludo e do pescoço \\
\hline C43.5 & Melanoma maligno do tronco \\
\hline C43.6 & Melanoma maligno do membro superior, incluindo ombro \\
\hline C43.7 & Melanoma maligno do membro inferior, incluindo quadril \\
\hline C43.8 & Melanoma maligno invasivo da pele \\
\hline C43.9 & Melanoma maligno de pele, não especificado \\
\hline Códigos & Outros tipos de neoplasias malignas da pele (C44) \\
\hline C44.0 & Neoplasia maligna da pele do lábio \\
\hline C44.1 & Neoplasia maligna da pele da pálpebra, incluindo o canto \\
\hline C44.2 & Neoplasia maligna da pele da orelha e do ducto auditivo externo \\
\hline C44.3 & Neoplasia maligna da pele de outras partes e partes não especificadas da face. \\
\hline C44.4 & Neoplasia maligna da pele do couro cabeludo e do pescoço \\
\hline C44.5 & Neoplasia maligna da pele do tronco \\
\hline C44.6 & Neoplasia maligna do membro superior, incluindo ombro \\
\hline C44.7 & Neoplasia maligna do membro inferior, incluindo quadril \\
\hline C44.8 & Neoplasia maligna de pele sobreposta \\
\hline C44.9 & Neoplasia maligna da pele, não especificado \\
\hline
\end{tabular}

A série de óbitos foi modelada utilizando o modelo autorregressivo integrado de média móvel, isto é, um ARIMA ( $p, d, q)$, conhecido também como modelo de Box-Jenkins (BOX; JENKINS; REINSEL, 1994), para o ajuste do número trimestral de óbitos por câncer de pele entre 1996 e 2012, assim como na previsão de valores trimestrais futuros.

Os procedimentos realizados seguem a seguinte ordem:

I) Análise gráfica da série original para verificar a presença de tendência e/ou sazonalidade;

II) Realização do teste Dickey-Fuller Aumentado (ADF) na série original, ao nível de significância de 5\%, para verificar a hipótese de que a série apresenta raiz unitária, ou seja, não é estacionária. Caso a série não seja estacionária, devese realizar diferenças sucessivas (d) nas observações até se obter estacionariedade;

III) Após atingir a estacionariedade da série, analisou-se o gráfico da Função de Autocorrelação (FAC) e da Função de Autocorrelação Parcial (FACP) como auxílio para obtenção dos modelos candidatos ajustados à série; 
IV) A partir dos modelos encontrados, utilizou-se o Critério de Informação de Akaike (AIC), o Erro Quadrático Médio de Previsão (EQMP) e o Erro Percentual Médio Absoluto (MAPE) como método de seleção do modelo com melhor ajuste e previsão. Escolheu-se aquele com menor valor de AIC, EQMP e MAPE;

V) Realizou-se o teste de Ljung-Box nos resíduos da série, ao nível de significância de 5\%, para verificar a adequação do modelo escolhido. Esse teste verifica se os resíduos do modelo estimado são estimativas do ruído branco, isto é, se não existe autocorrelação. Caso exista autocorrelação nos resíduos deve-se buscar outro modelo até que seja observado ruído branco. Também realizou-se o teste de Adequação do Qui-quadrado para a normalidade dos resíduos, assim como a análise gráfica do ajustamento através do Q-Q Plot;

VI) Apresentaram-se as previsões e os intervalos de confiança, ao nível de significância de 5\%, para os quatro trimestres de 2013, com a finalidade de validação do modelo preditivo, comparando-se os valores previstos com os valores reais. Se os intervalos de confiança contiverem esses valores concluise que a série pode ser utilizada para previsão do número de óbitos de trimestres futuros.

A fim de obter dados que subsidiaram a discussão foram calculadas as taxas de mortalidades brutas e ajustadas, o número médio de anos potenciais de vida perdidos e a variação percentual anual por sexo, através do Atlas on-line de mortalidade (ATLAS ON-LINE DE MORTALIDADE, 2015). As análises foram realizadas através do software livre Gnu Regression, Econometrics and Times-Séries Library (Gretl), versão $1.10 .90 \mathrm{cvs}$ (GRETL, 2015).

\section{RESULTADO}

No gráfico 1 está apresentada a série do número trimestral de óbitos por câncer de pele ocorridos entre 1996 e 2012, na Região Sul do Brasil, o que pode-se observar uma possível tendência de crescimento no número de casos ocorridos. 
Gráfico 1 - Série Temporal do número trimestral de óbitos por câncer de pele, ocorridos entre 1996 e 2012, na Região Sul do Brasil

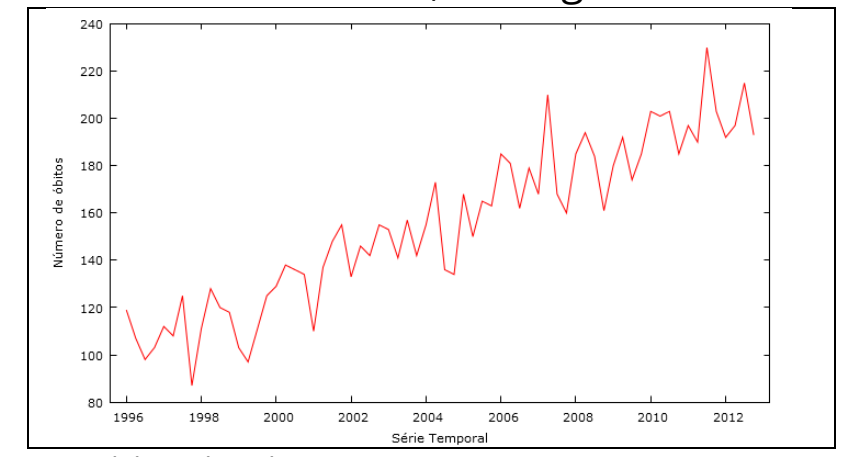

Fonte: Elaborado pelo autor.

No gráfico 2, através das funções de autocorrelação (FAC) e autocorrelação parcial (FACP), observa-se um comportamento de autocorrelação entre as defasagens, evidenciando a não-estacionariedade da série. Essa evidência pode ser comprovada através do resultado do Teste Dickey-Fuller Aumentado, que apresentou valor $-\mathrm{p}=0,9970$ para a série sem a presença da constante, e valor $-\mathrm{p}=0,0944$ para a série com a presença da constante. Portanto, não se rejeita a hipótese de que a série tenha raiz unitária ao nível de significância de 5\%, logo há a necessidade da realização de diferenças sucessivas na série original até torná-la estacionária.

Gráfico 2 - FAC e FACP da série do número trimestral de óbitos por câncer de pele na região Sul considerando as 25 primeiras defasagens

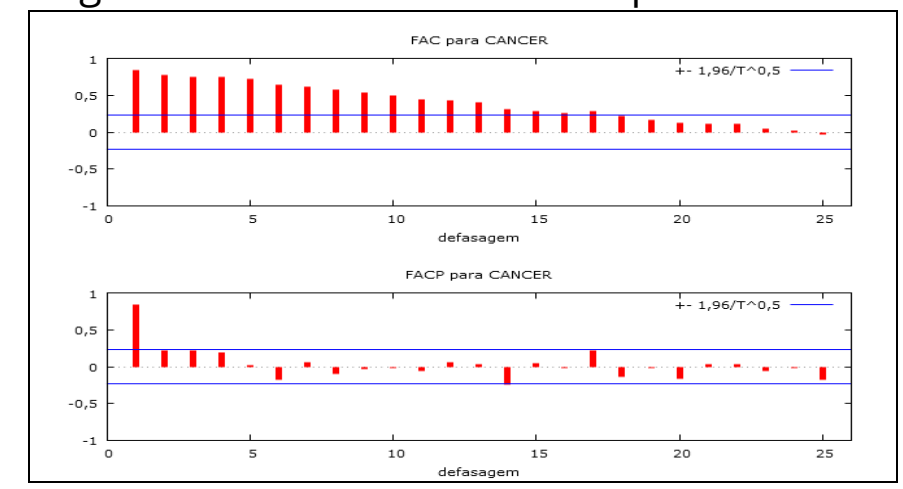

Fonte: Elaborado pelo autor.

A série em primeira diferença, ou seja, integrada de ordem 1, apresentada na Figura 3, sugere visualmente uma possível estacionariedade do período analisado. Por isso, realizou-se novamente o Teste Dickey-Fuller Aumentado, o qual apresentou valor $-\mathrm{p}=0,000$ para a série sem a presença da constante, e valor- $\mathrm{p}=0,000$ para a série com a presença da constante. Portanto, rejeita-se a hipótese de que a série tenha raiz 
unitária ao nível de significância de 5\%, assim sendo, a série tornou-se estacionária após a integração de ordem 1.

Gráfico 3 - Série Temporal diferenciada de ordem 1 do número trimestral de óbitos por câncer de pele, ocorridos na Região Sul do Brasil, entre 1996 e 2012

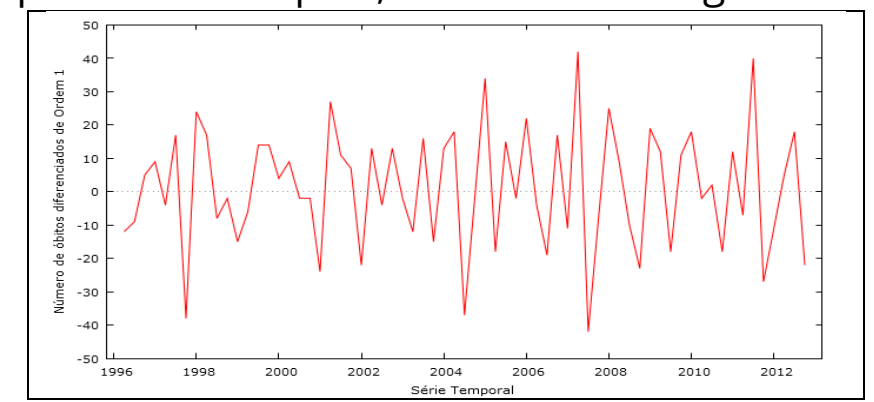

Fonte: Elaborado pelo autor.

Observa-se, através do gráfico 4, que existe defasagens fora dos limites de confiança, ou seja, significativos ao nível de significância de 5\%. A FAC apresentou a primeira defasagem fora dos limites de confiança, sugerindo a utilização de um modelo de média móvel de ordem 1. Já a FACP apresenta as quatro primeiras defasagens fora dos limites de confiança, sugerindo a utilização de um modelo autorregressivo de ordem 4. Portanto, tem-se um modelo ARIMA $(4,1,1)$ como possível ajuste da série. Porém, foi identificado que este não apresentou significância dos parâmetros, sendo necessário encontrar outros modelos de ajustamento.

Gráfico 4 - FAC e FACP da série diferenciada de ordem 1 do número trimestral de óbitos por câncer de pele na região Sul, considerando as 25 primeiras defasagens

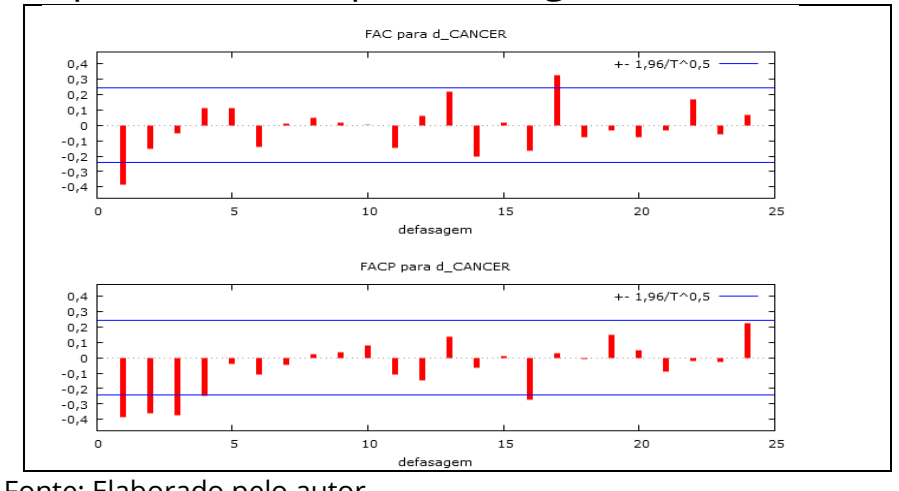

Fonte: Elaborado pelo autor.

A partir disso foram testados diversos modelos com e sem a presença da constante, e verificaram-se menores valores de AIC, EQMP e MAPE àqueles com o uso da constante, os quais estão apresentados na Tabela 1. Quanto aos dois modelos descritos, identificou-se que o ARIMA $(4,1,0)$ obteve tanto menor valor de AIC, quanto 
menores valores de EQMP e MAPE. Portanto, entre os modelos mostrados, este é o que melhor descreve o comportamento da série trimestral de óbitos por câncer de pele, na Região Sul brasileira, assim como o que melhor prevê períodos futuros, pois todos parâmetros foram significativos ao nível de significância de 5\%.

Tabela 1 - Modelos ajustados e seus critérios de seleção de modelo para séries diferenciadas de ordem 1

\begin{tabular}{lccccc}
\hline Modelos Ajustados & Parâmetros & Valor-p & AIC & EQMP & MAPE \\
\hline ARIMA(3,1,0) & constante $=1,3982$ & 0,0321 & 554,7639 & 198,3200 & 8,0331 \\
& $p_{1}=-0,6904$ & 0,0000 & & & \\
& $p_{2}=-0,5876$ & 0,0000 & & & \\
& $p_{3}=-0,3911$ & 0,0005 & & & \\
\hline ARIMA $(4,1,0)$ & constante $=1,4300$ & 0,0034 & 551,2253 & 182,1800 & 7,6489 \\
& $p_{1}=-0,8078$ & 0,0000 & & & \\
& $p_{2}=-0,7560$ & 0,0000 & & & \\
& $p_{3}=-0,5894$ & 0,0000 & & & \\
& $p_{4}=-0,2828$ & 0,0152 & & & \\
\end{tabular}

Fonte: Elaborado pelo autor.

Quanto a adequação do modelo, obteve-se pelo teste de Ljung-Box valor $-\mathrm{p}=0,6418$, o que indica que os resíduos são ruído branco, logo, pode-se considerar que esse possui uma boa adequação para a série, o que pode ser verificado no Gráfico 5.

Gráfico 5 - FAC e FACP dos Resíduos do modelo $\operatorname{ARIMA}(4,1,0)$ para a Série trimestral de óbitos

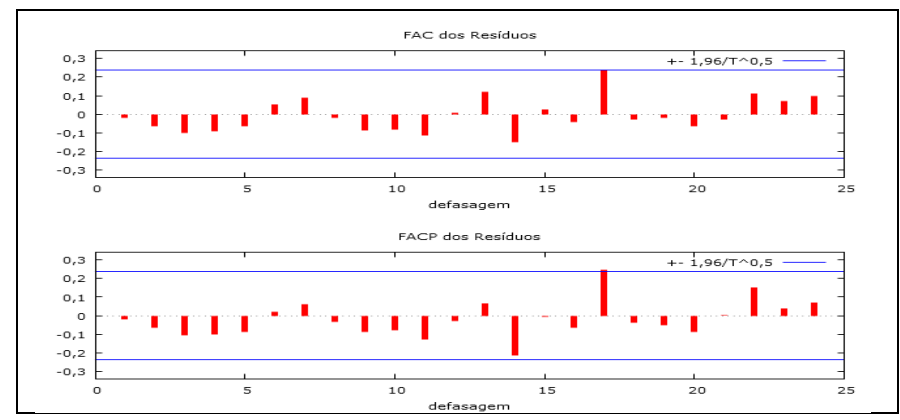

Fonte: Elaborado pelo autor.

Observou-se pelo Gráfico 6 que, existe evidência de normalidade nos resíduos, assim como o teste de adequação do Qui-quadrado comprovou essa hipóteses obtendo valor $-\mathrm{p}=0,6506$. 
Gráfico 6 - Q-Q Plot da Normalidade dos Resíduos do modelo ARIMA $(4,1,0)$ para os resíduos da série

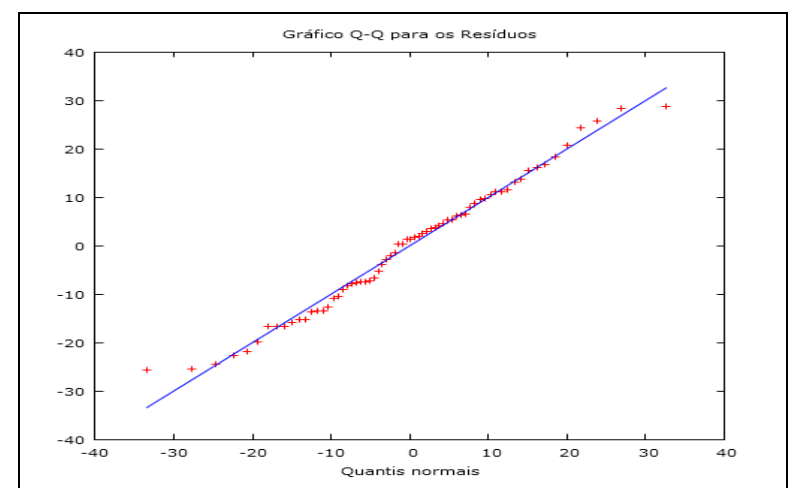

Fonte: Elaborado pelo autor.

Quanto as previsões, pode-se observar através do Gráfico 7 que os óbitos previstos pelo modelo apresentaram comportamento bastante parecido com os reais.

Gráfico 7 - Óbitos observados e Previsões de óbitos por Câncer de Pele de indivíduos, pelo modelo ARIMA $(4,1,0)$

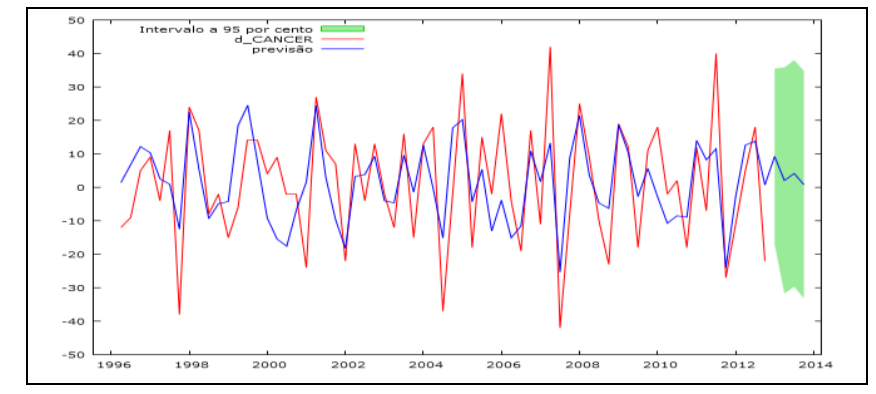

Fonte: Elaborado pelo Autor

Por fim, verificou-se que os intervalos de confiança, ao nível de significância de $5 \%$, contiveram os números de óbitos observados, validando, assim, o modelo preditivo.

Tabela 2 - Óbitos Observados, Previsões e Intervalo de confiança de 95\% do modelo ARIMA $(4,1,0)$ para os Quatro trimestres de 2013

\begin{tabular}{llllc}
\hline Períodos & Óbitos Observados & Previsões & Intervalo de Confiança das Previsões \\
\hline $1^{\circ}$ Trimestre & 219 & 202,24 & 175,97 & 228,51 \\
$2^{\circ}$ Trimestre & 211 & 204,30 & 177,55 & 231,05 \\
$3^{\circ}$ Trimestre & 218 & 208,44 & 181,59 & 235,29 \\
$4^{\circ}$ Trimestre & 227 & 209,23 & 181,90 & 236,55 \\
\hline
\end{tabular}

Fonte: Elaborado pelo autor. 


\section{DISCUSSÃO}

Estudos internacionais evidenciam que nas últimas décadas, em praticamente todos os países/regiões onde existem dados divulgados, há uma tendência de aumento da incidência do melanoma cutâneo (ARNOLD; et al., 2013; JEMAL; et al., 2011) e do número de óbitos por câncer de pele do tipo melanoma e não-melanoma.

O Modelo de Box-Jenkins melhor ajustado para os óbitos por câncer de pele não melanoma e melanoma no período estudado foi o ARIMA $(4,1,0)$, sendo um modelo com poder preditivo que estima para o primeiro trimestre de 2015 um número de 215 óbitos por esses tipos de câncer (IC95\%: 181-248), para o segundo trimestre 215 óbitos (IC95\%: 181-250), para o terceiro trimestre 218 óbitos (IC95\%: 182-253), e para o último trimestre 219 óbitos (IC95\%: 183-255), seguindo a tendência crescente evidenciada tanto à nível nacional quanto mundial.

A limitação deste estudo é inerente ao fato de ser um estudo observacional, e, por usar dados secundários de precisão e validade de variáveis, e que podem ser inadequados e/ou incompletos, mas somente é de caráter exploratório.

A avaliação de tendências de mortalidade e alertas quanto às taxas de incidência por cânceres de pele melanoma e não melanoma, quando produzidas por um registro de câncer de qualidade, podem ser utilizadas nas avaliações de necessidades e/ou aprimoramento de programas de prevenção e detecção precoce implantados na área de cobertura desses registros, sendo que avaliações desse tipo dão subsídios à criação de estratégias de controle do câncer, dessa forma, diminuindo o impacto da mortalidade por esta neoplasia, sobre os casos totais de cânceres (INSTITUTO NACIONAL DE CÂNCER, 2010).

O câncer de pele não-melanoma é o tipo de câncer mais frequente no Brasil em ambos os gêneros, para 2014 foram estimados 34990 novos casos, sendo que este, raramente é fatal e podem ser removidos cirurgicamente e/ou obter sua remissão quando detectado prematuramente. Já o câncer de pele melanoma apresenta letalidade elevada, porém sua incidência é baixa, sendo estimado 1800 novos casos para 2014, sendo que para a Região Sul 63\% foram desses dois tipos para esse último ano do gênero masculino. Levando-se em consideração todos os novos casos de 
cânceres, os de pele correspondem a 32\% dos casos novos, e essa região, comparada ao restante do país é a que apresenta as maiores taxas de incidência (ATLAS ON-LINE DE MORTALIDADE, 2015). Em função do subdiagnóstico e da subnotificação, pode-se ter, consequentemente, estimativas mais baixas das taxas de incidência e dos números esperados de casos novos do que realmente deveria ser totalizado (QUINN; PERKINS, 2010; INSTITUTO NACIONAL DE CÂNCER JOSÉ ALENCAR GOMES DA SILVA, 2014).

As taxas brutas de mortalidade específica, por 100000 habitantes, do melanoma maligno de pele e outras neoplasias de pele para o Brasil, em 2013, foi de 1,55 para o gênero masculino e 1,09 para o gênero feminino, seguindo um padrão aproximado ao da Taxa Padronizada Mundial que para o Gênero masculino foi de 1,79 e para o feminino de 1,03; a Taxa padronizada para o Brasil ficou em torno de 1,99 para o gênero masculino e 1,19 para o feminino. Considerando apenas a região Sul do país, verificase uma taxa ainda mais elevada de óbitos, obtendo-se para o Estado do Paraná taxa bruta de 2,33 para sexo masculino e 1,73 para o sexo feminino, para o Estado de Santa Catarina taxa bruta de 3,17 para o sexo masculino e 2,13 para o sexo feminino, e para o Estado do Rio Grande do Sul taxa bruta de 3,31 para o sexo masculino e 2,12 para o sexo feminino. Isto acarreta em uma Taxa de Anos Potenciais de Vida Perdidos para todas as faixas etárias entre 1996 e 2013, de 0,16 para os homens, e 0,10 para as mulheres, com limite superior de idade de 70 anos (ATLAS ON-LINE DE MORTALIDADE, 2015).

A Variação Percentual Média Anual (KLEINBAUM; KUPPER; MILLER; NIZAM, 1998) que avalia as tendências de indicadores de saúde, no caso da mortalidade, para os anos de 1996 a 2013 para os homens fica em torno de um acréscimo de 1,11\% e para as mulheres de $0,71 \%$, para os casos da Região Sul, sendo que considerou-se que a tendência foi de crescimento linear nesse intervalo de tempo (ATLAS ON-LINE DE MORTALIDADE, 2015), salientando-se a importância de estudos estatísticos de previsão para o embasamento de medidas preventivas junto à população.

Estudos ecológicos de séries temporais podem mostrar a evolução de números brutos e taxas específicas de doenças numa determinada população geograficamente definida, além de avaliar o impacto de intervenções na área da saúde, sendo, portanto, 
um delineamento adequado para se examinar tendências de coeficientes e mortalidade ao longo do tempo (FRANÇA JÚNIOR; MONTEIRO, 2000; MORGENSTERN, 1998).

O Câncer de pele está intimamente ligado à exposição solar, e essa tem efeito cumulativo na pele do indivíduo, por isso não basta apenas difundir nas primeiras décadas de vida o conhecimento sobre os efeitos dos fatores de risco na expectativa média de vida da população e, principalmente, sobre a qualidade de vida, pois é preciso ainda que se desenvolvam estratégias preventivas que envolvam diversos setores da sociedade, em prol da mudança de modos de vida baseada em evidências e de natureza duradoura.

Além disso, identificar indivíduos precocemente expostos a fatores de risco de natureza ambiental ou genética e intervir nesses grupos específicos pode contribuir para a redução da morbimortalidade por cânceres malignos de pele.

\section{CONCLUSÃO}

Os objetivos da pesquisa foram atingidos e obtiveram-se as seguintes conclusões:

- O modelo ARIMA $(4,1,0)$ apresentou melhor ajuste dentre os modelos testados para explicar o comportamento da série trimestral do número de óbitos por câncer de pele, ocorridos na região Sul do Brasil, entre 1996 e 2012.

- Como consequência da validação do modelo para os dados trimestrais de 2013 tem-se um modelo preditivo para o número de óbitos trimestrais por câncer de pele nesta região.

- Por fim, este estudo evidenciou a necessidade de intervenções e medidas de prevenção em nível de políticas públicas, junto à população, visando à redução dos óbitos por essas causas, pois este estudo constatou que há um aumento do número de óbitos por câncer de pele ao longo do tempo. 


\section{REFERÊNCIAS}

ARNOLD, M.; HOLTERHUES, C.; HOLLESTEIN, L. M.; COEBERGH, J. W. W. et al. Trends in incidence and predictions of cutaneous melanoma across Europe up to 2015. J. Eur. Acad. Dermatol. Venereol., 2013.

ATLAS ON-LINE DE MORTALIDADE. Instituto Nacional de Câncer José Alencar Gomes da Silva. 2015. Disponível em: https://mortalidade.inca.gov.br/MortalidadeWeb/. Acesso em: jul. 2015

BOX, G. E. P.; JENKINS, G. M.; REINSEL, G. C. Times series analysis: forecasting and control, $3^{a}$ Ed. San Francisco: Holden-Day, 1994.

DATASUS, Estatísticas Vitais. Disponível em:

http://datasus.saude.gov.br/informacoes-de-saude/tabnet/estatisticas-vitais. Acesso em: nov. 2014.

FRANÇA JÚNIOR, I.; MONTEIRO, C. A. Estudo da tendência secular de indicadores de saúde como estratégia de investigação epidemiológica. Rev Saúde Pública 2000; 34 Supl 6:57.

GRETL. Gnu Regression, Econometrics and Times-Séries Library (Gretl), versão 1.10.90cvs. Disponível em: http://gretl.sourceforge.net/. Acesso em: jul. 2015.

INSTITUTO NACIONAL DE CÂNCER. Registros hospitalares de câncer: planejamento e gestão. Instituto Nacional de Câncer. 2 ed. - Rio de Janeiro: INCA, 2010.

INSTITUTO NACIONAL DE CÂNCER JOSÉ ALENCAR GOMES DA SILVA. Coordenação Geral de Ações Estratégicas. Coordenação de Prevenção e Vigilância. Estimativa 2014: incidência de câncer no Brasil. Rio de Janeiro: Instituto Nacional de Câncer; 2014. Disponível em: http://www.inca.gov.br/estimativa/2014/estimativa-24042014.pdf. Acesso em: nov. 2014.

JeMAL, A.; SARAIYA, M.; PATEL, P.; CHERELA, S. S. et al. Recent trends in cutaneous melanoma incidence and death rates in the United States, 1992-2006. J. Am. Acad. Dermatol., v. 65, n. 5, suppl 1, p. S17.e1-S17.e11, 2011.

JEMAL, A. et al. Global cancer statistics. CA: a cancer journal for clinicians, 2011, 61(2): 69-90.

KLEINBAUM, D. G.; KUPPER, L. L.; MILLER, K. E.; NIZAM, A. Applied regression analysis and multivariable methods. 3rd Ed. Pacific Grove: Duxbury Press; 1998.

MARKS, R.; KOPF, A. W. Cancer of the skin in the next century. International journal of dermatology, v. 34, n. 7, p. 445-447, 1995. 
MORGENSTERN H. Ecologic studies. In: Rothman KJ;Greenland S. 2nd ed. Modern epidemiology. Philadelphia: Lippincott-Raven Publishers; 1998.

QUINN, A. G.; PERKINS, W. Non-melanoma skin cancer and other epidermal skin tumors. In: Burns T, Breathnach S, Cox N, Griffiths C, editors. Rook's textbook of dermatology. 8th ed. Oxford: Blackwell Publishing; 2010. p. 52.1.

RHODES, A.R. et al. Risk factors for cutaneous melanoma: a pratical method of recognizing individuals. J. Amer. med. Ass., 285: 3146-54, 1987.

SBD, Programa Nacional de Controle do Câncer da Pele. Rio de Janeiro. Disponível em: http ://www.sbd.org.br/acoes/programa-nacional-de-combate-ao-cancerda-pele. Acesso em: nov. 2014. 